Evi dence for a Tor oi dal - Monent um Tr ansport Nondi ffusi ve Termfrom the JFT-2M Tokanak

\begin{tabular}{|l|l|}
\hline $\begin{array}{l}\text { j our nal or } \\
\text { publ i cat i on ti t l e }\end{array}$ & Physi cal Revi ew Let ter s \\
\hline vol une & Vol. 74 \\
\hline number & No. 11 \\
\hline page range & pp. 1990-1993 \\
\hline year & 1995- 03 01 \\
\hline URL & ht t p: //hdl . handl e. net /10655/122 \\
\hline
\end{tabular}




\title{
Evidence for a Toroidal-Momentum-Transport Nondiffusive Term from the JFT-2M Tokamak
}

\author{
K. Ida, Y. Miura,* T. Matsuda,* K. Itoh, S. Hidekuma, S.-I. Itoh ${ }^{\dagger}$ and JFT-2M Group* \\ National Institute for Fusion Science, Nagoya 464-01, Japan
}

(Received 22 February 1994)

\begin{abstract}
Toroidal rotation velocity profiles are measured by multichord charge-exchange spectroscopy for discharges where the direction of the neutral beams is changed from parallel to the plasma current to antiparallel. Analysis of the transport of toroidal momentum in the transient phase suggests the existence of a nondiffusive term in the transport matrix. This nondiffusive term results in a spontaneous source of toroidal momentum in the direction antiparallel to the plasma current.
\end{abstract}

PACS numbers: $52.25 . \mathrm{Fi}, 52.50 . \mathrm{Gj}$

An important issue in the study of the transport properties of plasmas is the evaluation of the transport coefficients for the particle, momentum, and heat fluxes. The transport matrix has nine coefficients for the particle, momentum, and energy fluxes; the diagonal elements are the diffusive terms, consisting of particle diffusivity $D$, viscosity $\mu$, and thermal diffusivity $\chi$, while the offdiagonal terms are considered the nondiffusive terms. Knowledge of the nondiffusive terms is required in order to understand the physics of anomalous transport. With respect to the particle flux, the concept of significant particle pinch (inward convective velocity) was found to be necessary to explain the peak electron-density profiles that are observed in Ohmic tokamak plasmas, since the particle source is localized near the plasma edge [1].

In [2], the particle pinch observed in the JAERI Fusion Torus-2M (JFT-2M) tokamak, with neutral beam injection antiparallel to the plasma current, was compared with the inward pinch model related to the electric field. Only the diffusive terms of heat or momentum flux associated with the heat and momentum transport, however, have been discussed in most transport analyses [3-5]. This is because the heat or momentum deposition is more or less localized near the plasma center in most discharges, and a simple diffusive model can only explain the measured temperature or rotational velocity profiles in a steady state.

Recently, the existence of a nondiffusive term for the electron heat flux (inward heat flux) was demonstrated in the off-axis electron cyclotron heating $(\mathrm{ECH})$ experiment in DIII-D [6]. The off-axis neutral beam injection experiments in the tokamak fusion test reactor (TFTR) have shown however that the measured toroidal rotational velocity profiles can be reproduced without introducing momentum pinch [7]. To date, one report has been published suggesting a nondiffusive term in the momentum transport [8]. The present Letter discusses our experimental results for the time evolution of toroidal rotational velocity profiles. Analysis of the transport of toroidal momentum during the transient phase, where the toroidal rotational velocity changes sign due to the changing of the direction of the injected neutral beam, suggests the existence of a nondiffusive term in the toroidal momentum transport. This term results in spontaneous plasma rotation in a direction antiparallel to the plasma current.

The JFT-2M is a medium size tokamak $(R=1.3 \mathrm{~m}$, $a=0.35 \mathrm{~m}$ ). The hydrogen neutral beams (NBs) are injected at an injection energy of $32 \mathrm{keV}$ from parallel (coinjection, $P=0.49 \mathrm{MW}$ ) to antiparallel (counterinjection, $P=0.56 \mathrm{MW}$ ) to the plasma current, or counterinjection to coinjection in order to study the response of the toroidal rotational velocity [2]. A series of experiments were performed under conditions of a $1.3 \mathrm{~T}$ toroidal field, a plasma current of $240 \mathrm{kA}$ with deuterium as the working gas, a limiter configuration, an elongation of 1.2. The profiles of the toroidal rotation velocity $v_{\phi}$ and ion temperature $T_{i}$ were measured by multipoint charge-exchange spectroscopy (CXS) every $16.6 \mathrm{~ms}$, using Carbon VI (CVI $529.05 \mathrm{~nm}, \Delta n=8-7$ ) charge-exchange line emission [9]. Since the spectra for all channels were measured with one charge-coupled device (CCD) detector, the offset of the wavelength channels to channel is small, being within a half pixel, i.e., $0.005 \mathrm{~nm}$. This corresponds to a velocity of $3 \mathrm{~km} / \mathrm{s}$ being much smaller than the central velocities $v_{\phi}$ of $50 \mathrm{~km} / \mathrm{s}$ (cophase) and $-100 \mathrm{~km} / \mathrm{s}$ (counterphase). Corrections for toroidal rotation due to the energy-dependent charge-exchange excitation rate [10] are required for high-temperature plasmas such as those found in the TFTR [11] and JET [12]. The ion temperature in the JFT-2M, however, is small enough $\left(T_{i}=0.5-0.7 \mathrm{keV}\right)$ to ignore these effects. The apparent velocity due to this effect is less than $3-4 \mathrm{~km} / \mathrm{s}$, and always towards the beam (codirection for the counter-NBI phase and counterdirection for the co-NBI phase). Therefore, $v_{\phi}$ is slightly underestimated due to the effect of energy-dependent charge-exchange excitation. This effect, however, does not result in an offset of $v_{\phi}$ because, in the present experiments, the beams are interchanged while the direction of the plasma current is kept fixed.

Figure 1 displays profiles of toroidal momentum $\left(m_{i} n_{i} v_{\phi}\right)$ for discharges when the direction of the injected neutral beam changes from co to counter [Fig. 1(a)] and from counter to co [Fig. 1(b)] to the plasma current at the flat top of the plasma current $(t=750 \mathrm{~ms})$. Here, $m_{i}$ is the ion mass and $n_{i}$ is the ion density of a deuteron. 
In steady-state toroidal rotation, the density profiles are measured by 13-channel Thomson scattering before $(t=690 \mathrm{~ms})$ and after $(t=890 \mathrm{~ms})$ the interchange of the neutral-beam injection. The measured profiles are well fitted by a parabolic-shaped profile raised to a power, $n_{e}(\rho)=n_{e}(0)\left(1-\rho^{2}\right)^{\alpha}$, where $\alpha=0.9-1.6$. During the transient phase, the electron density profiles are determined by two fitting parameters, $n_{e}(0)$ and $\alpha$, given by two line-averaged densities measured at $\rho=0.24$ and 0.6 by a multichord far infrared (FIR) laser interferometer.

The $m_{i} n_{i} v_{\phi}$ profiles at $t=742 \mathrm{~ms}$ (Fig. 1) are considered to be initial steady-state profiles prior to the onset of the transient phase. The toroidal rotation reaches almost steady-state values at $t=825 \mathrm{~ms}(75 \mathrm{~ms}$ after the interchange of NB). The electron density gradually increases, as $n_{e}(0)$ goes from $3.5 \times 10^{19} \mathrm{~m}^{-3}$ to $4.4 \times$ $10^{19} \mathrm{~m}^{-3}$, and $\alpha$ goes from 0.9 to $1.1,50 \mathrm{~ms}$ after the interchange of NB from coinjection to counterinjection. On the other hand, the electron density decreases, as $n_{e}(0)$ decreases from $6.7 \times 10^{19} \mathrm{~m}^{-3}$ to $6.1 \times 10^{19} \mathrm{~m}^{-3}$ and $\alpha=$ $1.6 \rightarrow 1.5,50 \mathrm{~ms}$ after the change of NB from counterinjection to coinjection. The change of electron density, however, within the time resolution of the CXS measurements $(16.6 \mathrm{~ms})$, is $3 \%-7.5 \%$, and results in only a small contribution to the change of toroidal momentum dur-
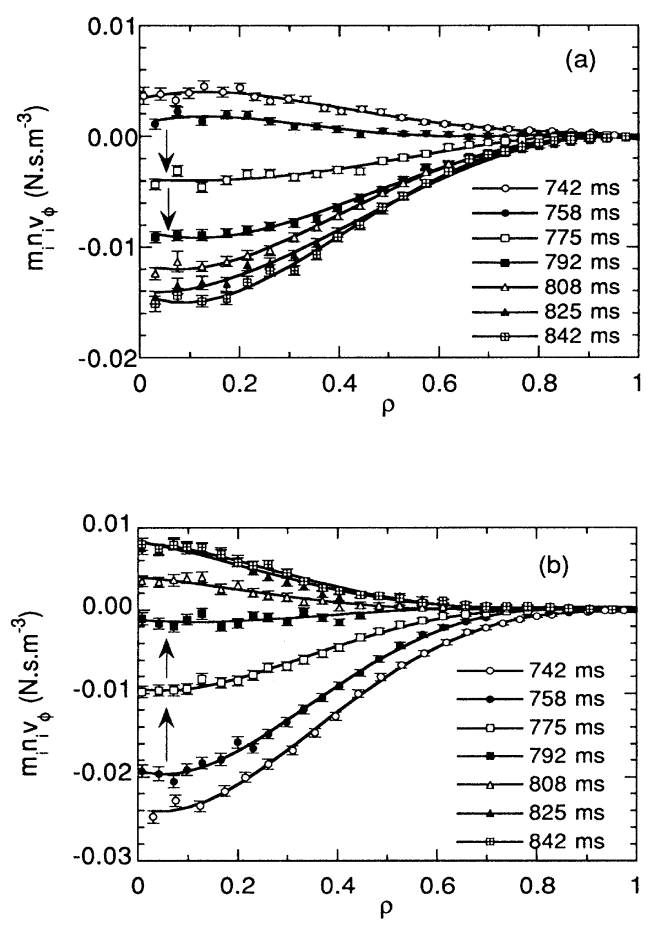

FIG. 1. Radial profiles of toroidal momentum during the transient phase for discharges when the direction of the NBI is reversed. (a) The coinjection is activated from 550 to $750 \mathrm{~ms}$ and the counterinjection from 750 to $950 \mathrm{~ms}$. (b) The counterNBI is activated from 550 to $750 \mathrm{~ms}$ and the co-NBI from 750 to $950 \mathrm{~ms}$. The various measurements were performed according to the figure legends. ing the transient phase. The central electron temperature, measured with a soft $\mathrm{x}$-ray pulse height analyzer (PHA), remains nearly constant $[0.95 \mathrm{keV}]$ in time, while the ion-temperature profiles measured at $t=783 \mathrm{~ms}$ are $0.53\left(1-\rho^{2}\right)^{1.9}+0.08$ and $0.66\left(1-\rho^{2}\right)^{2.4}+0.08[\mathrm{keV}]$ for the coinjections to counterinjections and counterinjections to coinjections, respectively. The soft $\mathrm{x}$-ray signal exhibits sawtooth oscillations in the cophase, which disappears $100 \mathrm{~ms}$ after the onset of counterinjection. No other significant MHD activities which change plasma confinement are observed. The toroidal momentum in the counterinjection steady-state phase is two to three times greater than that in the coinjection phase, although the injected NB power during coinjection is comparable to that during counterinjection. There are two mechanisms to explain the larger momentum during the counterinjection phase. One is the reduction of momentum viscosity, the other the existence of another source of momentum, not driven by the neutral beam, termed a spontaneous source of momentum in this article. The analysis of transport in the transient phase yields the momentum viscosity and the spontaneous source of toroidal momentum, while analysis of the steady state can give only the momentum viscosity.

The radial flux of toroidal momentum can be estimated from the time derivative of toroidal momentum and the toroidal force driven by NBI as $\Gamma_{M}(r)=$ $(1 / r) \int\left\{\partial\left(m_{i} n_{i}(r) v_{\phi}(r)\right] / \partial t-f_{n b i}(r)\right\} r d r$. Here, $f_{n b i}(r)$ is the toroidal force calculated by a Monte Carlo beam deposition code where positive values define the force in the direction of coinjection. Interactions with impurity ions are included in this code, but the effect of plasma rotation on the deposition and thermalization of the beam [13] is ignored, since the toroidal rotation velocity is less than $4 \%$ of the beam ion velocity. The parallel and perpendicular nonthermal beam ion energies are $9.1 \%$ and $10.8 \%$ of the total stored energy for coinjection, and $6.2 \%$ and $7.7 \%$ for counterinjection, respectively. The contributions of nonthermal ions to the total momentum are then $8.3 \%$ for coinjection and $3.4 \%$ for counterinjection.

The prompt deposition of momentum into the plasma is also taken into account [14]. The momentum deposition profiles for counter-NBI are broader than those of co-NBI, due to the drift orbit motion of beam ions. When the momentum flux $\Gamma_{M}(r)$ is proportional to the gradient of $m_{i} n_{i} v_{\phi}$, the momentum transport is considered to be purely diffusive in nature. The measured toroidal momentum profiles are well fitted by a fifth-order polynomial (6 parameters) with multiple-correlation coefficients of $0.96-0.99$. Their gradients are derived from the coefficients of the polynomial. By subtracting and averaging values measured at two nearby time slices, the momentum flux and toroidal momentum gradient are evaluated, as shown in Fig. 2, for averaged minor radii. The error bars due to the uncertainties in beam deposition $(\sim 10 \%)$ and in the gradient of $m_{i} n_{i} v_{\phi}$ are also plotted. These figures clearly show the finite offset of momentum flux at the zero gradient of toroidal momentum for $\rho$ less than 
0.6. This pinch term vanishes near the plasma axis where $\rho$ approaches zero. This spontaneous momentum flux is found to be negative (the counter direction) both for coinjection [Fig. 2(b)] and counterinjection [Fig. 2(a)]. The toroidal momentum flux contributed by the neutral-beamdriven force $f_{\mathrm{NBI}}$ at $\rho=0.6$ is $-0.016 \mathrm{~N} \mathrm{~m}^{-2}$ for counterinjection, and $0.017 \mathrm{~N} \mathrm{~m}^{-2}$ for coinjection. Spontaneous momentum flux is observed as being $\frac{1}{5}-\frac{1}{2}$ of the beamdriven momentum flux in the core region. The toroidal rotation velocity in the Ohmic phase can be evaluated by measurements immediately after the neutral beam injection, or by the short NBI pulse $(\Delta t<10 \mathrm{~ms})$, since the toroidal momentum confinement time is relatively long $(>30 \mathrm{~ms})$. The velocity is always in the counter direction $\left[v_{\phi}(0)=-20--30 \mathrm{~km} / \mathrm{s}\right]$, regardless the direction of the NBI. The results are consistent with toroidal rotation in the counter direction for an Ohmic plasma in other tokamaks (see, for instance, the results from the PDX [15]) and for a plasma with a perpendicular NBI in the JIPP T-IIU tokamak [16]. The "intrinsic toroidal rotation" is determined by the balance between the spontaneous source of momentum and the momentum viscosity. One candidate for the source of the spontaneous momentum is the $\mathbf{j} \times \mathbf{B}$ force due to the fast ion orbit loss. In these dis-
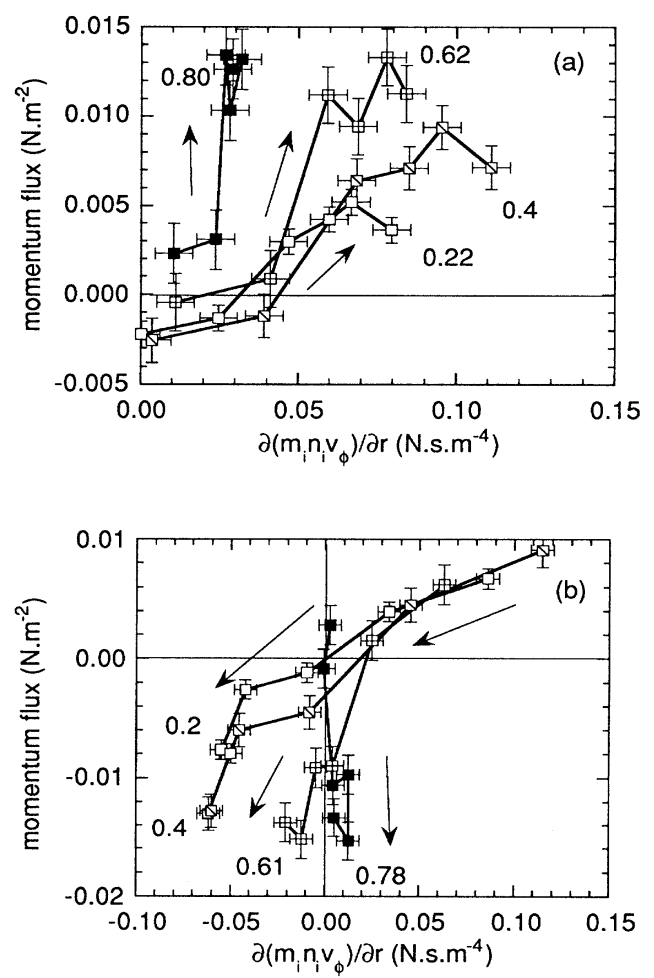

FIG. 2. Radial fluxes of toroidal momentum as a function of the gradient of toroidal momentum for the discharges when the direction of the NBI is reversed (a) from co to counter, and (b) from counter to co for various averaged minor radii. The six points for each radius represent the measured values at $t=767,783,800,817,833$, and $850 \mathrm{~ms}$. charges, the calculated ion orbit loss is negligible (1\%) for coinjection, while a significant fraction $(11 \%)$ is expected for counterinjection. The observed spontaneous source of the momentum in coinjection, however, is comparable to that found during counterinjection. If the ion orbit loss is the cause of the spontaneous momentum flux, it should increase towards the plasma edge. The measured spontaneous momentum flux, however, does not exhibit such a trend. The existence of a spontaneous pinch in the Ohmic plasma suggests that the NBI is not the origin for the spontaneous momentum flux. It can be concluded then that the ion orbit loss is not the main cause of the spontaneous momentum flux.

The theory which has been developed for anomalous viscosity due to drift-wave turbulence predicts that the momentum flux is generated by the density and/or iontemperature gradient, as well as the velocity gradient [17]. For simplicity, ignoring the momentum flux generated by the density gradient, the observed spontaneous term is assumed to be driven only by the gradient of ion temperature. This is also supported by the observations that both the pinch term and $\nabla T$ vanish as $\rho$ approaches zero. The radial momentum flux is then given by $\Gamma_{M}=\mu_{\phi} \partial\left(m_{i} n_{i} v_{\phi}\right) / \partial r+\mu_{\phi / \Delta T}\left(v_{\mathrm{th}} / T_{i}\right) \partial\left(m_{i} n_{i} T_{i}\right) / \partial r$. Here, $v_{\text {th }}$ is the thermal velocity, while $\mu_{\phi}$ and $\mu_{\phi / \Delta T}$ are the respective diagonal and off-diagonal coefficients for momentum transport. The momentum viscosity $\mu_{\phi}$ and the off-diagonal coefficient were derived from the slope and offset of the six time sliced data, measured at 767 , $783,800,816,832$, and $850 \mathrm{~ms}$. The radial profiles of these coefficients are shown in Fig. 3. The error bars indicated in Fig. 3 are the statistical error bars determined by fitting the six data points with a line, including the statistical and systematic uncertainties in the values of the data points. The data indicated as co to counter (solid lines) stand for the momentum viscosity estimated during the counterinjection phase $16-100 \mathrm{~ms}$ after the neutral beams are interchanged. The magnitude of the momentum viscosity $\mu_{\phi}$ during the counterinjection phase is almost identical to that in the coinjection phase, while the toroidal rotational velocity and toroidal momentum during the counterinjection are 2 to 3 times greater than those in coinjection. Here, analysis of the steady state at $t=$ $842 \mathrm{~ms}$ and $\rho=0.4$ under the assumption of no spontaneous momentum source yields a momentum viscosity $\mu_{\phi}$ of $0.22 \mathrm{~m}^{2} / \mathrm{s}$ for coinjection and $0.08 \mathrm{~m}^{2} / \mathrm{s}$ for counterinjection. The reduction of momentum viscosity can be expected well after the neutral beams are changed from coinjection to counterinjection due to the electron density being peaked, as seen in the effective thermal diffusivity ( $\chi_{\text {eff }}=0.96 \mathrm{~m}^{2} / \mathrm{s} \rightarrow 0.54 \mathrm{~m}^{2} / \mathrm{s}$ at $\rho=0.4$ ) [2]. The density peaking, however, is relatively slow (100 to $150 \mathrm{~ms}$ ) compared with the change in toroidal rotational velocity. Therefore, it is not difficult to understand that the momentum viscosity presently estimated in the transient phase does not exhibit any reduction during the counterinjection phase. The off-diagonal coefficients $\mu_{\phi} / \Delta T$ estimated 

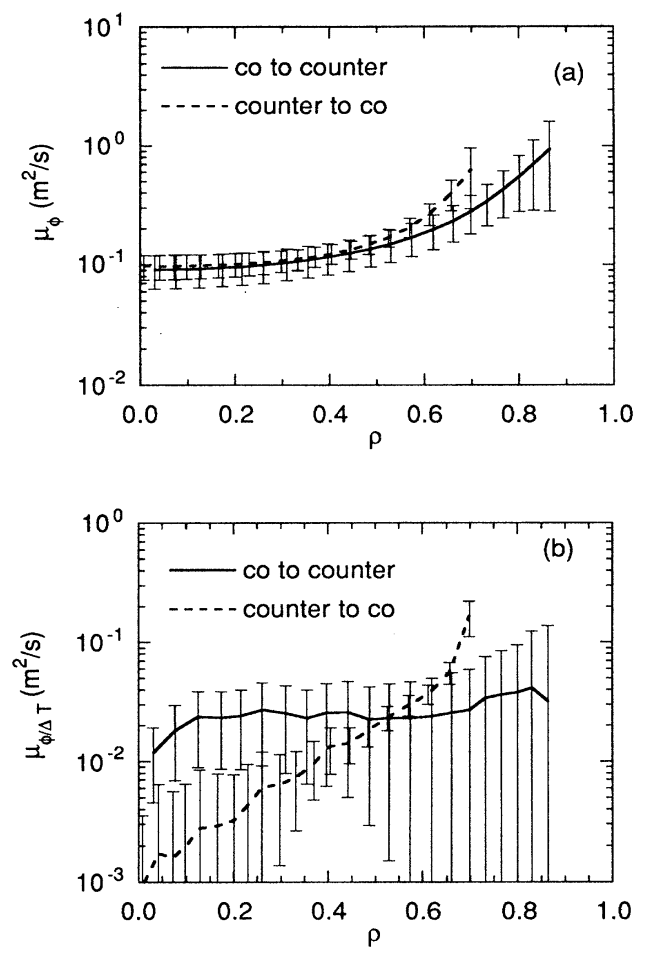

FIG. 3. Radial profiles of the (a) momentum viscosity and (b) off-diagonal coefficient derived from the six time sliced data of the toroidal-momentum fluxes and gradients shown in Fig. 2.

from the offset of the measured momentum flux and the ion temperature gradient are on the order of $10^{-2} \mathrm{~m}^{2} / \mathrm{s}$ for these discharges. It should be noted that the off-diagonal term is not very small compared with the diagonal term, since it should be multiplied by $v_{\mathrm{th}} / v_{\phi}[=2-5]$ to compare it with $\mu_{\phi}$.

In the analysis described here, it has been assumed that the bulk toroidal rotational velocity is identical to that of carbon measured with the CXS, since there are no measurements of bulk toroidal rotation in the JFT$2 \mathrm{M}$. Here, it was estimated how much of a velocity difference $\Delta v_{\phi}$ between carbon and bulk ion can be expected. According to neoclassical theory [18], carbon impurities rotate more in the counterdirection than bulk ions by $8 \mathrm{~km} / \mathrm{s}$. The offset of toroidal rotational velocity evaluated in the present transport analysis, however, is $-25 \mathrm{~km} / \mathrm{s}$. It is noted that if $\Delta v_{\phi}$ is almost constant in space and time, then its effect on the gradient of $n_{i} m_{i} v_{\phi}$ and the momentum flux should be small. Therefore, the nondiffusive momentum flux discussed in the present Letter cannot be explained by the neoclassical effects. To confirm the spontaneous momentum source measured in the JFT-2M, more measurements of other impurities and bulk plasma, if possible, are required. Measurements of toroidal rotational velocity on the other side (the high field side of the magnetic axis) should be conducted in the future to confirm the effect of the Pfirsch-Schluter ion flow [19]. It should be emphasized that the transient behavior of carbon toroidal rotation during the interchange of neutral beams observed in the JFT-2M cannot be explained by a pure diffusive transport model, suggesting the possibility of an off-diagonal, or at least a nondiffusive, term of momentum transport. The correlation of spontaneous momentum with the $T_{i}$ gradient presented as an example would provide an order-of-magnitude estimate of the offdiagonal term to be used in theoretical studies. The experimental discrimination of the influences due to the gradients of $n_{i}$ and $T_{i}$ requires further research.

The authors wish to thank M. Kojima for help in data acquisition and Dr. M. Mori, Dr. N. Suzuki, and the NBI and JFT-2M machine operation groups for their support during this experiment. Thanks are also extended to Dr. K. Tani (JAERI) for making available the numerical code of NB deposition. The authors also thank Dr. M. Fujiwara and Dr. T. Hamada for their continuous encouragement. The paper is dedicated to the memory of Dr. Hikosuke Maeda (JAERI).

*Japan Atomic Energy Research Institute, Naka-machi, Naka-gun, Ibaragi 319-11, Japan.

${ }^{\dagger}$ Research Institute for Applied Mechanics, Kyushu University, Kasuga 816, Japan.

[1] B. Coppi, Comments Plasma Phys. Controlled Fusion 5, 261 (1980).

[2] K. Ida et al., Phys. Rev. Lett. 68, 182 (1992).

[3] K. H. Burrell et al., Nucl. Fusion 28, 3 (1988).

[4] H. Weisen et al., Nucl. Fusion 29, 2187 (1989).

[5] A. Kallenbach et al., Nucl. Fusion 30, 645 (1990).

[6] T. C. Luce, C. C. Petty, and J.C. M. deHaas, Phys. Rev. Lett. 68, 52 (1992).

[7] R. J. Hawryluk et al., Plasma Phys. Controlled Fusion 33, 1509 (1991)

[8] K. Nagashima et al., Nucl. Fusion 34, 449 (1994).

[9] K. Ida and S. Hidekuma, Rev. Sci. Instrum. 60, 867 (1989).

[10] R. J. Fonck et al., Phys. Rev. A 29, 3288 (1984).

[11] R. B. Howell et al., Rev. Sci. Instrum. 59, 1521 (1988).

[12] M. G. von Hellermann et al., Rev. Sci. Instrum. 61, 3479 (1990).

[13] R. J. Goldston, in Proceedings of the Workshop on Basic Physical Processes of Toroidal Fusion Plasmas, Varenna, 1985 (CEC, Brussels, 1986), Vol. 1, p. 165.

[14] F. L. Hinton and J. A. Robertson, Phys. Fluids 27, 1243 (1984).

[15] K. Brau et al., Nucl. Fusion 23, 1643 (1983).

[16] K. Ida et al., Nucl. Fusion 31, 943 (1991).

[17] S.-I. Itoh, Phys. Fluids B4, 796 (1992), J. Phys. Soc. Jpn. 59, 3431 (1990).

[18] Y. B. Kim et al., Phys. Fluids B3, 2050 (1991).

[19] M. C. Zarnstorff and S. C. Prager, Phys. Rev. Lett. 53, 454 (1984). 